\title{
A RECIRCULATING LINAC-BASED FACILITY FOR ULTRAFAST X-RAY SCIENCE*
}

\author{
J. N. Corlett, W. A. Barletta, S. DeSantis, L. Doolittle, W. M. Fawley, M.A. Green, P. Heimann, S. Leone, S. Lidia, \\ D. Li, A. Ratti, K. Robinson, R. Schoenlein, J. Staples, W. Wan, R. Wells, A.Wolski, and A. Zholents, LBNL, \\ Berkeley, California, USA; F. Parmigiani, UCSC, Brecia, Italy; M. Placidi, W. Pirkl, Geneva, Switzerland; \\ R. A. Rimmer, Thomas Jefferson National Accelerator Facility, Virginia, U.S.A., S. Wang, Indiana University, U.S.A.
}

\begin{abstract}
We present an updated design for a proposed source of ultra-fast synchrotron radiation pulses based on a recirculating superconducting linac [1,2], in particular the incorporation of EUV and soft $\mathrm{x}$-ray production. The project has been named LUX - Linac-based Ultrafast X-ray facility. The source produces intense $\mathrm{X}$-ray pulses with duration of $10-100 \mathrm{fs}$ at a $10 \mathrm{kHz}$ repetition rate, with synchronization of 10's fs, optimized for the study of ultra-fast dynamics. The photon range covers the EUV to hard x-ray spectrum by use of seeded harmonic generation in undulators, and a specialized technique for ultra-shortpulse photon production in the 1-10 keV range. Highbrightness rf photocathodes produce electron bunches which are optimized either for coherent emission in freeelectron lasers, or to provide a large $\mathrm{x} / \mathrm{y}$ emittance ration and small vertical emittance which allows for manipulation to produce short-pulse hard x-rays. An injector linac accelerates the beam to $120 \mathrm{MeV}$, and is followed by four passes through a 600-720 MeV recirculating linac. We outline the major technical components of the proposed facility.
\end{abstract}

\section{OVERVIEW}

A recirculating linac accelerates 2 ps electron bunches to $2.5-3 \mathrm{GeV}$, where the bunches radiate in multiple insertion devices. Intense soft $\mathrm{x}$-rays are produced by cascaded harmonic generation scheme, similar to high-gain harmonic-generation (HGHG) - a laser-seeded process in a cascaded series of undulators, resulting in enhanced radiation at selected harmonics of the seed $[3,4]$. The coherent soft $\mathrm{x}$-rays can be tuned over a range of tens of $\mathrm{eV}$ to $1 \mathrm{keV}$, and ultrashort seed laser pulses produce pulse durations of 10-200 fs. Hard x-rays are produced by spontaneous emission of the electrons in narrow-gap, short-period undulators. By use of a novel bunch tilting process followed by optical compression, hard x-ray pulse durations of 50-100 fs are obtained over a range of 1-10 $\mathrm{keV}[1,5]$. Synchronization of the $\mathrm{x}$-rays with lasers is critical for experiments, and optical pulses initiate both cascaded harmonic generation seed lasers and experimental end station amplifiers for precise timing $[1,6]$. The femtosecond x-rays are produced at a $10 \mathrm{kHz}$ repetition rate, with variable polarization, and with peak fluxes comparable to third generation light sources. Thus, the

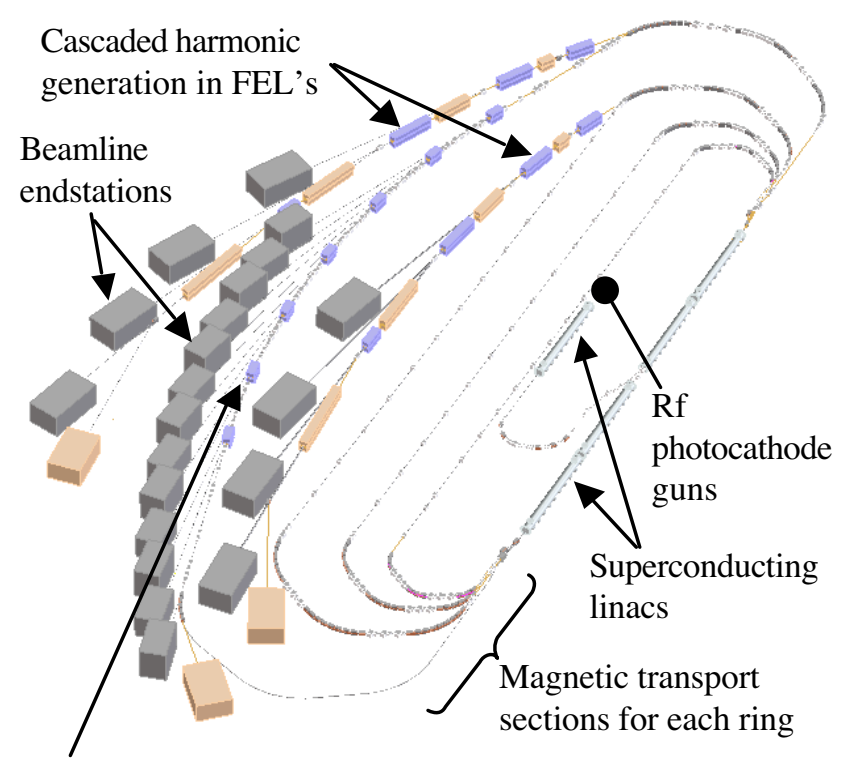

Hard x-ray production in narrow-gap

short-period insertion devices

Figure 1. Machine layout. The beams generated at the rf photocathode guns travel through the injector linac, main linac, transport arcs, deflecting cavities, and either hard $\mathrm{x}$ ray production section or a cascaded harmonic generation section, to the beam dump. Machine footprint is approximately $150 \times 50 \mathrm{~m}$.

proposed LUX facility would provide stable, synchronized, tunable, ultrafast $\mathrm{x}$-ray pulses to multiple beamlines operating simultaneously over a broad range of $\mathrm{x}$-ray wavelengths.

\section{ACCELERATOR DESIGN}

Electron pulses are produced at a rate of $10 \mathrm{kHz}$ in highbrightness rf photocathode guns $[7,8]$. Two sources are used - one with a conventional circular cross-section beam optimized for production of high-brightness EUV and soft $\mathrm{x}$-ray radiation in the harmonic generation scheme, the other with a flattened cross-section beam for production of hard $\mathrm{x}$-rays [8]. The beam quality requirements of the rf photocathode guns are already demonstrated, with normalized emittance of approximately $3 \mathrm{~mm}$-mrad at 1 nC charge, although higher repetition rates have not been addressed to date. Conventionally, rf photocathode guns employ a simple right-circular geometry or "pillbox"

\footnotetext{
* This work supported by the U.S. Department of Energy under Contract No. DE-AC03-76SF00098
} 
shaped cavities, and operate over $10-100 \mathrm{~Hz}$ pulse repetition rate. The cathode is mounted on the cavity axis and electrons are rapidly accelerated by the rf electric fields, minimizing space-charge effects in the low-energy beam. For high duty cycle operation, thermal limitations prevent such designs from operating at an electric field sufficiently high to produce good beam emittance. For the LUX facility we have produced a conceptual design optimized for operation at high gradient and high repetition rate, and producing low-emittance bunches. This design incorporates features that increase cavity surface area to reduce deposited power density and enhance the accelerating electric field at the cathode $\left(82 \mathrm{~W} / \mathrm{cm}^{2}\right.$ maximum power density for $64 \mathrm{MV} / \mathrm{m}$ at the cathode) [7].

Application of a solenoidal magnetic field on the cathode of the flat-beam gun, followed by a specially configured skew-quadrupole channel located after the injector linac, allows production of a "flat" beam with $\mathrm{x} / \mathrm{y}$ emittance ratio 50/1 and small vertical normalized emittance of $0.4 \mathrm{~mm}$-mrad $[8,9]$. This technique has been successfully demonstrated at Fermilab, with properties close to LUX design parameters [10].

Following the injector linac, a third-harmonic cavity is used to linearize the correlated energy spread introduced in the injector linac, and to manipulate the longitudinal phase-space in preparation for bunch compression. The beam is then transported to the entrance of the recirculating linear accelerator. In the transport line from injector linac to main linac, the bunches are compressed from 20 ps to 2 ps, with a final energy spread of \pm 200 $\mathrm{keV}$. This transport line is carefully designed to compensate for the effects of coherent synchrotron radiation at the shorter bunch lengths [11].

In the recirculating linac the maximum energy of 3 $\mathrm{GeV}$ is achieved after four passes through the $720 \mathrm{MeV}$ superconducting rf structure, or $2.5 \mathrm{GeV}$ with the main linac operating at $600 \mathrm{MeV}$. Identical cryomodules containing multiple accelerating cavities are used for the main linac and the injector linac. The superconducting linacs have advantages in providing a compact and efficient accelerator, extremely stable rf fields, and inherently small perturbative effects on the beam. Significant advances have been made in superconducting $\mathrm{rf}$ technology in recent years, and the parameters of the proven TESLA superconducting rf systems developed at DESY have been used in LUX design studies [12]. Planned upgrades for the CEBAF facility at TJNAF also meet the requirements for the LUX linacs [13]. Our design is for an accelerating gradient of up to $20 \mathrm{MV} / \mathrm{m}$ in the main linac. The electron bunch repetition interval in LUX is less than the superconducting cavity filling time, and the linacs are more efficiently operated in $\mathrm{cw}$ mode. Engineering modifications to existing cryomodule designs required to accommodate significantly increased thermal load in the liquid helium are described in [1].

The flexibility of the LUX lattice design allows control and preservation of electron beam transverse and longitudinal emittances, minimizing the influence of collective effects [11,14]. Longitudinal and transverse dynamics have been modeled from the RF gun through the injector linac and all passes of the main linac. In the injector, a harmonic cavity will be used to control the longitudinal phase-space following the injector linac [11]. The bunch length and magnet bend angle in the lowest energy arcs of the machine result in a regime in which coherent synchrotron radiation emission could be expected, and the vacuum chamber geometry is designed to minimize this effect by shielding against lower-frequency radiation [15]. The recirculating ring arcs are achromatic and isochronous to preserve beam quality. Our studies include particle tracking with cavity wakefields, resistive wall impedance, magnet errors and misalignments, and show only modest emittance growth, with negligible impact on machine performance. The lattice is designed to allow manipulation of the bunch phase space on each pass if required, and also to accommodate bunch rates greater than the $10 \mathrm{kHz}$ baseline design.

LUX will have the capacity for energy recovery in the linacs. However, for the baseline beam power of a few tens of $\mathrm{kW}$, the beam will be taken directly to a shielded dump following the $\mathrm{x}$-ray production sections.

At the exit of the final arc the flat-beam electron bunches receive a time-correlated vertical kick in a dipolemode RF cavity. This imparts to the electron bunch a transverse momentum that is correlated in amplitude to longitudinal position within the bunch. The electrons then radiate $\mathrm{x}$-rays in the downstream chain of undulators and dipole magnets, imprinting this correlation in the geometrical distribution of the x-ray pulse. The correlated $\mathrm{x}$-ray pulse is then compressed to 10's fs duration by use of asymmetrically cut crystal optics. The bunch deflecting technique is identical to the "crab-cavity" schemes proposed for several electron-positron colliders. A total deflecting voltage of $8.5 \mathrm{MV}$ is required, and we have developed a preliminary design for a 7-cell superconducting deflecting cavity [16].

Narrow-gap in-vacuo superconducting undulator designs provide tunable high-flux sources in the $1-12 \mathrm{keV}$ range. The calculated flux of $10 \mathrm{keV}$ photons from $1 \mathrm{nC}$ bunches at $3 \mathrm{GeV}$ and $10 \mathrm{kHz}$ is $6 \times 10^{10}$ photons $/ \mathrm{s} / 0.1 \% \mathrm{BW}$ for a 4 $\mathrm{mm}$ gap, $14 \mathrm{~mm}$ period, $2 \mathrm{~T}$ peak magnetic field undulator. Similar insertion devices are currently being prototyped and designs are expected to mature in the near future.

A laser-seeded cascaded harmonic-generation scheme produces high-flux, short-pulse photons over an energy range of tens of $\mathrm{eV}$ to $1 \mathrm{keV}$. In this process the circular cross-section high-brightness electron beam is extracted from the recirculating linac, and passed through an undulator where a co-propagating seed laser modulates the charge distribution over a short length of the bunch. The scheme has been developed and demonstrated at the Brookhaven DUV FEL facility [4]. The imposed modulation results in enhanced radiation at specific 
wavelengths and a selected wavelength is amplified in a following undulator, tuned to a higher harmonic of the seed laser. The electron pulse is then delayed in a short chicane, and the process repeated by modulating a fresh portion of the beam this time with the harmonic radiation produced in the previous undulator. Using a tunable optical parametric amplifier as the seed, and variable undulators, allows significant tunability in four stages of harmonic generation, variable flux up to $10^{13}$ photons per pulse, and variable pulse duration depending on the seed laser parameters [3]. Two chains of cascaded harmonic generation are proposed, providing exceptional flexibility in producing EUV and soft $\mathrm{X}$-ray pulses. Circular polarization is attainable by use of elliptical undulators, and flux stability of $0.1 \%$ or better is obtained in seconds from random pulse-pulse flux variations of $10-20 \%$ at 10 $\mathrm{kHz}$ repetition-rate. The use of tapered undulators allows tailoring of flux to individual experiments, to avoid spacecharge effects in, for example, photoemission processes.

Sophisticated laser systems will be an integral part of the LUX facility, providing experiment excitation pulses, and stable timing signals, as well as the electron source through the photocathode laser. Each endstation will have it's own dedicated laser system and optical manipulation and diagnostics, and optical tables and equipment will be contained within a stable and controlled environment. Multiple tuneable lasers covering a range of 267-3000 nm and pulse durations of $\leq 50 \mathrm{fs}$ are required for experiment initiation, together with temporal and spatial filtering to optimize performance for specific experimental applications. Distribution systems using fibre-optic transmission lines will provide optical seed pulses to each beamline, with feedback based on interferometric measurements to stabilize the path lengths [6]. Developments in laser technology are expected to result in significant improvements in the coming years, which will be incorporated into our design with minimal impact on accelerator systems.

Synchronization and timing of the ultra-short x-ray pulses to the experimental excitation pulse is critical to studies of ultra-fast dynamics. For LUX we propose to generate inherently stable pulses by using seeded FEL systems and bunch manipulation. In the case of EUV and soft $\mathrm{x}$-ray production, the cascaded harmonic generation seed laser oscillator also drives the sample excitation laser, resulting in timing stability of approximately 20 fs. For our scheme of hard x-ray production by bunch manipulation followed by $\mathrm{x}$-ray pulse compression, we find that the phase jitter of the deflecting cavities with respect to the experimental laser pulse dominates timing issues [1]. Phase and amplitude feedback of the deflecting cavities is expected to provide $\mathrm{x}$-ray pulse to laser pulse timing stability of $50 \mathrm{fs}$ or better. To stabilize all timing and $\mathrm{rf}$ signals in the facility, we propose to use a phaselocked laser oscillator as the facility master oscillator. The RF gun, linacs, and deflecting cavities may thus be phaselocked to the experimental excitation lasers, and timing jitter between the optical laser and the x-ray pulse emitted by the beam minimized [6].

\section{SUMMARY}

A recirculating linac user facility is proposed to address the growing national and international need for ultrafast $\mathrm{x}$ ray scientific research. The LUX facility is based on existing accelerator technology, coupled with an array of advanced tunable femtosecond lasers, and is capable of performing an enormous variety of pump-probe type experiments with soft and hard x-rays. The facility has been specifically designed with a view toward solving problems in ultrafast science, and it's impact will be across all fields of science.

\section{REFERENCES}

[1] J. N. Corlett et al, "Feasibility study for a recirculating linac-based facility for femtosecond dynamics", LBNL formal report LBNL-51766, December 2002.

[2] J. N. Corlett, et al, " A Recirculating Linac Based Synchrotron Light Source for Ultrafast X-ray Science", Proc. EPAC'02, Paris, France, June 2002.

[3] W. Fawley et al, "Simulation studies of an XUV/soft $\mathrm{x}$-ray harmonic-cascade FEL for the proposed LBNL recirculating linac", this conference.

[4] L.-H. Yu et al, "High-Gain Harmonic-Generation FreeElectron Laser", Science 289 932-934 (2000).

[5] A. Zholents et al "Generation of subpicosecond x-ray pulses using RF orbit deflection", NIM A 425 (1999) 385-389.

[6] J. Corlett et al, "Techniques for Synchronization of Xray Pulses to the Pump Laser in an Ultrafast X-ray Facility", this conference.

[7] J. Staples et al, "The LBNL Femtosource $10 \mathrm{kHz}$ Photoinjector", this conference.

[8] S. Lidia et al, "An Injector for the Proposed Berkeley Ultrafast X-Ray Light Source", this conference.

[9] S. Wang et al, "Flat beam production in low energy injectors", this conference

[10] D. Edwards et al, "The Flat Beam Experiment at the FNAL Photoinjector", Proc. XXth International Linac Conference, Monterey, 2000.

[11] A. Zholents et al, "Longitudinal phase-space control in the Berkeley Femtosecond X-ray light source", this conference.

[12] TESLA Technical Design Report, DESY 2001-011, March 2001.

[13] L. Harwood, C. Reece, "CEBAF at 12 and $25 \mathrm{GeV",}$ Proc. SRF2001, Tsukuba, Japan, Sept. 2001.

[14] S. De Santis et al "Collective effects analysis for the Berkeley femtosource", this conference

[15] R. Wells et al, "Recirculating linac vacuum system", this conference

[16] D. Li et al, "Deflecting rf cavity design for a recirculating linac-based facility for ultrafast $x$-ray science", this conference. 